\title{
THE NUCLEAR AND AERIAL DYNAMICS OF THE TUNGUSKA EVENT
}

\author{
S. J. D. D'ALESSIO and A. A. HARMS \\ McMaster University, Hamilton, Ontario, Canada
}

(Received in final form 24 November 1988)

\begin{abstract}
A mathematical-physical characterization of an atmospheric "explosive" event-commonly called the Tunguska Event of 1908-has been formulated. Emphasis is placed upon the aerial dynamics and the nuclear energy released in the gas cap of the meteor as it passed through the atmosphere. The results obtained are consistent with the dominant phenomena observed for the Tunguska Event suggesting therefore a plausible reconstruction of the physical processes associated with this unusual event.
\end{abstract}

\section{INTRODUCTION}

On 30 June 1908, in Central Siberia of the U.S.S.R., an extraordinary event occurred (Oliver, 1928; Crowther, 1931). Eyewitnesses reported a giant fireball moving across the sky followed by an overpowering shockwave. Trees were radially toppled over thousands of square kilometers, seismic and atmospheric disturbances were recorded as far away as England, and the next several nights were sufficiently bright for reading. However, no significant impact crater was formed nor was any extraterrestrial matter found in the immediate area.

A number of hypotheses have been advanced as possible explanations for this so called Tunguska Event (named after a nearby river). Among these are cometary/meteoric burnup in the atmosphere (Whipple, 1930; Oberg, 1977; Florensky, 1963), matter-antimatter annihilation (Cowan et al., 1965), black-hole impact (Jackson and Ryan, 1973) and even alien intervention from outer space (Baxter and Atkins, 1976).

The physical evidence points most strongly to a massive meteor moving at hypersonic speed and burning up in the atmosphere. Indeed, the meteor is believed to be a small comet and the difference in terminology strictly refers to the origin of the moving object. Meteors originate from the asteroid belt while comets are believed to originate from the Oort cloud. The comet theory is substantiated by the observance of the body in the early morning hours which rules out a meteor as these objects generally impact the Earth in an overtaking orbit and thus would be seen in the afternoon hours. Comets, on the other hand, are believed to follow no favoured orbits and thus may collide with the Earth in either an overtaking or a head-on collision. By definition, a meteoroid or comet-or even an alien spacecraft-constitutes a meteor once it enters the atmosphere. Approximate estimates of the comet's mass and speed suggest that a high temperature, detached shockwave would form, possibly providing conditions for the fusion of deuterium nuclei supplied mostly by the ablative materials of the hydrogenous composition of the comet. Indeed, the chemical energy is insignificant when compared with the nuclear fusion energy, and hence, establishes a motive for pursuing a thorough investigation as to the amount of fusion energy liberated in the gas cap. One must remember that, although a nuclear reaction liberates much more energy than a chemical reaction, the conditions in the gas cap may favour chemical reactions to proceed at a much higher rate than the nuclear reactions, which can more than make up for the differences in energy release per reaction.

Though some useful geophysical data and eyewitness reports on this Tunguska Event are on record, considerable critical information of relevance to our analysis is absent. We judge, however, that cometary physics, the aerodynamics of high speed blunt bodies, and our understanding of fusion reactions, has advanced sufficiently to seek an additional, physically plausible elaboration on this Tunguska Event.

\section{INTERACTION DYNAMICS}

Some dominant features of the Tunguska Event may be reconstructed as follows. The evidence of seismic and atmospheric disturbances recorded, together with human observations, leave no doubt that an object of extraordinary kinetic energy interacted with the Farth's atmosphere. The absence of an enormous crater may suggest complete or nearly complete burnup and the appearance of several bright nights suggest that the comet's ashes and its tail interacted with the atmosphere over Asia and Europe. This 


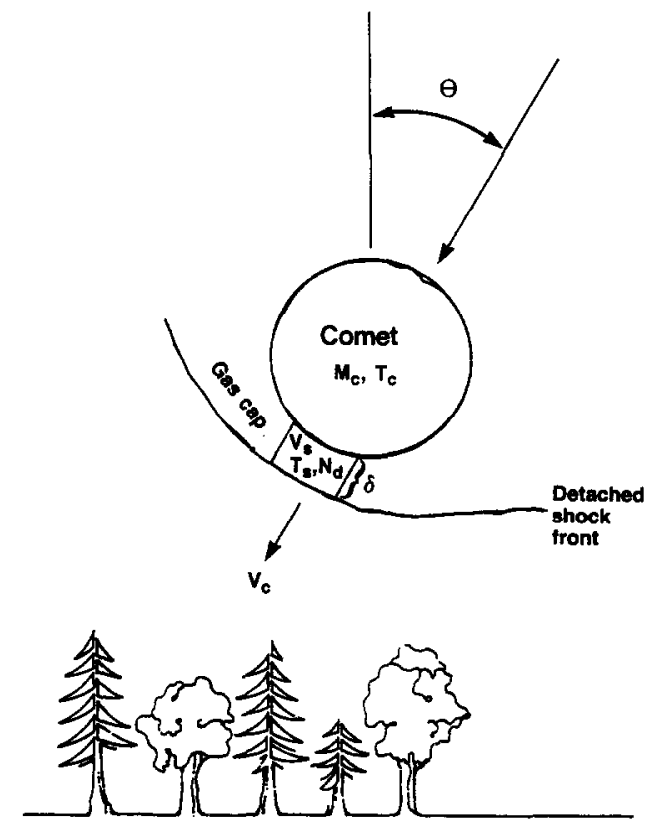

Fig. 1. Schematic OF THE TUNGUSKa COMETARY IMPACT. Here the symbols used represent the comet mass $\left(M_{\mathrm{c}}\right)$, comet mean temperature $\left(T_{c}\right)$, comet velocity $\left(V_{c}\right)$, zenith angle $(\theta)$, stagnation temperature $\left(T_{s}\right)$, stagnation volume $\left(V_{s}\right)$, deuterium density $\left(N_{\mathrm{d}}\right)$, and the stand-off distance $(\delta)$.

phenomenon is also in agreement with the comet hypothesis as the comet's tail points in the anti-solar direction which in this case would mean directly over Asia and Europe.

The present commonly accepted view is that a comet is a "dirty snowball" and therefore provides considerable amounts of hydrogen and hence the deuterium isotope. As the comet entered the atmosphere, a frontal shock wave was formed. The high temperature of the gas cap in front of the comet's leading edge caused evaporation and fragmentation of the comet's surface. Consequently, the deuterium density in the gas cap increased. The hypersonic flight of the comet ensured the formation of a detached shock wave with temperatures sufficiently high to consider fusion reactions occurring in the gas cap. For the brief duration of the comet's life in the atmosphere, the process in the leading edge may be characterized as a continuous explosive progression ceasing upon burnup or impact. That is, the Tunguska Event constitules a brief naturally operating fusion reactor with nature providing ignition by aerodynamic heating and confining the plasma in the gas cap of the comet.

In Fig. 1 we suggest, in schematic form, the dominant variables of interest. The comet possesses at some arbitrary time, a mass $M_{\mathrm{c}}$, mean temperature $T_{\mathrm{c}}$, speed $V_{\mathrm{c}}$ and stagnation volume $V_{\mathrm{s}}$. Because we are dealing with a blunt body, most of the incident energy received is transformed into aerodynamic heating of the gas cap as opposed to the heating of the body surface. Of interest to us here are the fusion reactions and mass-energy transfers in the stagnation volume of the gas cap.

The reason for our interest in the stagnation volume is that the analysis of the problem is tractable in this domain. For example, since the shock front formed in front of this volume is normal, well established relations involving temperature, pressure, and density can be invoked. Further, the leakage of material out of this stagnation volume can be modelled by the analogous fluid mechanics problem concerning the impingement of two opposed radial jets (Witze and Dwyer, 1976), one being the stream of air molecules entering the volume while the other is the ablating material from the comet surface. Further, it is known that under these conditions, the stagnation domain represents a volume of $\sim 0.02$ of the total gas cap volume (Freeman, 1956) and thus allows an upper estimate of the total nuclear energy release as the conditions for fusion are expected to be most favourable in this stagnation volume since the local temperature and density are highest. In this volume then, we are concerned with ion densities $N_{\mathrm{i}}$-for the i-type ions-existing at a kinetic temperature $T_{\mathrm{s}}$.

\section{NUCLEAR KINETICS}

The existence of dueterium, d, of density $N_{\mathrm{d}}$, in the stagnation volume provides for two concurrent equiprobable self-fusion reactions (Chen, 1974; Gill, 1981)

$$
\begin{aligned}
& \mathrm{d}+\mathrm{d} \rightarrow \mathrm{p}+\mathrm{t}+Q_{\mathrm{dd}, \mathrm{t}}, R_{\mathrm{dd}, \mathrm{t}} ; \\
& \mathrm{d}+\mathrm{d} \rightarrow \mathrm{n}+\mathrm{h}+Q_{\mathrm{dd}, \mathrm{h}}, R_{\mathrm{dd}, \mathrm{h}} .
\end{aligned}
$$

Here the symbols $\mathrm{p}, \mathrm{t}, \mathrm{n}$ and $\mathrm{h}$ represent a proton, triton, neutron and helium-3, respectively; $Q_{\mathrm{dd}, \mathrm{t}}$ and $Q_{\mathrm{dd}, \mathrm{h}}$ are the reaction $Q$-values; $R_{\mathrm{dd}, \mathrm{t}}$ and $R_{\mathrm{dd}, \mathrm{h}}$ are the reaction rate densities given by Harms (1987):

$$
\begin{aligned}
R_{\mathrm{dd}, \mathrm{t}} & =\frac{N_{\mathrm{d}}^{2}(t)}{2}\langle\sigma v\rangle_{\mathrm{dd}, \mathrm{t}}, \\
R_{\mathrm{dd}, \mathrm{h}} & =\frac{N_{\mathrm{d}}^{2}(t)}{2}\langle\sigma v\rangle_{\mathrm{dd}, \mathrm{h}},
\end{aligned}
$$

with $\{\sigma v\}_{\mathrm{dd},()}$ as the corresponding reaction rate parameters depending only upon the kinetic temperature of the deuterium population (McNally et al., 1979).

The instantaneous rate of nuclear energy released 
in a unit stagnation volume is therefore

$$
\begin{aligned}
\frac{\mathrm{d} E_{\mathrm{s}}}{\mathrm{d} t} & =R_{\mathrm{dd}, \mathrm{t}} Q_{\mathrm{dd}, \mathrm{t}}+R_{\mathrm{dd}, \mathrm{h}} Q_{\mathrm{dd}, \mathrm{h}} \\
& =N_{\mathrm{d}}^{2}(t)\left[\frac{\langle\sigma v\rangle_{\mathrm{dd}, \mathrm{t}} Q_{\mathrm{dd}, \mathrm{t}}+\langle\sigma v\rangle_{\mathrm{dd}, \mathrm{h}} Q_{\mathrm{dd}, \mathrm{h}}}{2}\right] .
\end{aligned}
$$

The determination of the nuclear power thus generated in this volume requires knowledge of the deuterium density with time, $N_{\mathrm{d}}(t)$, as well as the kinetic temperature of these ion populations so as to specify $\{\sigma v\}_{(\}}$. Since $\langle\sigma v\rangle_{\mathrm{dd}, \mathrm{t}} \approx\langle\sigma v\rangle_{\mathrm{dd}, \mathrm{h}}$, we take $\langle\sigma v\rangle_{\mathrm{dd}}=\langle\sigma v\rangle_{\mathrm{dd}, \mathrm{t}}=\langle\sigma v\rangle_{\mathrm{dd}, \mathrm{h}}$ (McNally et al., 1979). Thus equation (3) reduces to

$$
\frac{\mathrm{d} E_{\mathrm{s}}}{\mathrm{d} t}=\bar{Q}_{\mathrm{dd}} N_{\mathrm{d}}^{2}(t)\langle\sigma v\rangle_{\mathrm{dd}}
$$

where $\bar{Q}_{\mathrm{dd}}$ represents the average of $Q_{\mathrm{dd}, \mathrm{t}}$ and $Q_{\mathrm{dd}, \mathrm{h}}$.

Further, the deuterium ion density must satisfy the following rate equation

$$
\frac{\mathrm{d} N_{\mathrm{d}}}{\mathrm{d} t}=\frac{\beta_{\mathrm{d}}}{V_{\mathrm{s}}}\left|\frac{\mathrm{d} M_{\mathrm{c}}}{\mathrm{d} t}\right|\left(1-L_{\mathrm{f}}\right)-N_{\mathrm{d}}^{2}(t)\langle\sigma v\rangle_{\mathrm{dd}} .
$$

Here, we included its supply rate by comet ablation and its loss by self-fusion and leakage; $\beta_{\mathrm{d}}$ is the deuterium fraction ablating into the stagnation volume and $L_{\mathrm{f}}$ is the normalized leakage factor as discussed and defined in the Appendix.

\section{AERIAL DYNAMICS}

In order to solve equations (4) and (5), it is necessary to specify $\mathrm{d} M_{\mathrm{c}} / \mathrm{d} t$ as well as the stagnation temperature $T_{\mathrm{s}}$. The assumed quasistatic temperature in the stagnation volume is given approximately by the solution of the following energy balance equation (Goulard, 1964)

$$
c_{\mathrm{p}} T_{\mathrm{sf}}+\frac{V_{\mathrm{e}}^{2}}{2}=c_{\mathrm{p}} T_{\mathrm{s}}+\frac{(\gamma-1) c^{2}}{4 \gamma}+\frac{\sigma T_{\mathrm{s}}^{4}}{\rho_{\mathrm{a}} V_{\mathrm{c}}}
$$

where $c_{\mathrm{p}}$ is the specific heat of the gas at constant pressure, $\gamma$ is the ratio of specific heats, $c$ is the speed of sound, $\sigma$ is the Stefan-Boltzmann constant, $\rho_{\mathrm{a}}$ is the air density and $T_{\mathrm{sf}}$ is the temperature at the shock front. For an isothermal atmosphere the air density varies as

$$
\rho_{\mathrm{a}}=\rho_{0} \mathrm{e}^{-z / h}
$$

with $\rho_{0}$ as the sea level atmospheric density and $h$ as the scale height. The shock front temperature is that predicted by the Rankine-Hugoniot relations (Anderson, 1984) so that for hypersonic flight, as is our case, this temperature can be formulated as

$$
T_{\mathrm{sf}}=\frac{2 T_{\mathrm{c}} \gamma(\gamma-1)}{(\gamma+1)^{2}}\left(\frac{V_{\mathrm{c}}}{c}\right)^{2}
$$

where $T_{\mathrm{e}}$ is the surrounding average atmospheric temperature.

The solution of equation (6) represents the aerodynamic temperature directly behind a steady, normal shock front. This corresponds to the quasistatic approximation as the temperature is assumed to deviate infinitesimally from equilibrium values or along the comet trajectory as it passed through the atmosphere. This also corresponds to the temperature imposed by nature onto the stagnation volume. Because the gas cap is optically thick, ${ }^{*}$ the temperature, $T_{\mathrm{s}}$, can be taken to be spatially independent within the stagnation volume. This is a very good approximation in the interior of the stagnation volume; however, near the shock front and the comet surface there exist thin boundary layers where the temperature gradients are extremely high as shown in Fig. 8. Because these layers are much less than the stand-off distance, $\delta$, only a small negligible fraction of the gas resides there. This is equivalent to the following interpretation. As mass ablates from the comet surface, it is blown across the thermal boundary layer into the interior of the stagnation volume where it quickly comes to equilibrium with the surrounding gas and is ready for fusion. The air stream entering through the shock front ensures that the ablated mass will remain in the interior of the stagnation volume. Again, this process can be viewed as the impingement of two directly opposing radial jets. Of the non-equilibrium processes taking place in the stagnation volume, ionization is the most important, imposing, however, no significant effect as the comet is travelling well in excess of the critical ionization velocity proposed by Alfvén and Arrhenius (1975).

Equations (6) and (8) demand that the comet velocity, $V_{c}$, be known during its flight through the atmosphere. Thus, an equation is required to state how $V_{c}$ changes. For this purpose we use

$$
\frac{\mathrm{d} V_{\mathrm{c}}}{\mathrm{d} t}=-\frac{\Gamma A \rho_{0} V_{\mathrm{c}}^{2} \mathrm{e}^{-z / h}}{M_{\mathrm{c}}^{1 / 2} \rho_{\mathrm{c}}^{2 / 3}}+g \cos \theta
$$

where $A$ is the shape factor, $\Gamma$ is the drag coefficient, $\theta$ is the zenith angle, $g$ is the average acceleration due to gravity, and $\rho_{c}$ is the density of the comet. Also, the altitude, $z$, varies according to

* By optically thick we mean that the photon mean free path is much less than $\delta$, the distance between the comet surface and the shock front, known as the stand-off distance (see Fig. 1). 


$$
\frac{\mathrm{d} z}{\mathrm{~d} t}=-V_{\mathrm{c}} \cos \theta
$$

Equation (9) is a statement of Newton's Second Law in which the first term on the right-hand side represents a deceleration brought about by the aerodynamic drag. While many other forces such as buoyancy, retro-rocket effect and electrostatic drag are present, our calculations reveal that they are relatively insignificant. Also, it is assumed that the Earth is "flat" and that $\theta$ remains constant; that is, the comet trajectory is a straight line. Both of these are excellent approximations for near perpendicular entries into the atmosphere, which is our domain of interest. For near horizontal entries, the curvature of the Earth must be taken into account as well as an equation to govern how the zenith angle, $\theta$, will vary.

Lastly, $\mathrm{d} M_{\mathrm{c}} / \mathrm{d} t$ needs to be specified ; for this we use

$$
\frac{\mathrm{d} M_{\mathrm{c}}}{\mathrm{d} t}=-\frac{A}{L}\left(\frac{M_{\mathrm{c}}}{\rho_{\mathrm{c}}}\right)^{2 / 3} Q
$$

This equation governs how the mass of the comet is changing. Here we are assuming that all the energy received by the comet surface is transformed into vaporizing its surface while very little is left to heat the body. In the case of a meteor entering the Earth's atmosphere, the mass remains fairly constant at first as the energy is going into heating the body. When the body reaches either its melting or boiling point, severe mass ablation sets in while its body temperature then remains fairly constant. In our case of a comet, severe vaporization commences well before it even enters the Earth's atmosphere due to solar heating, and thus, the mean body temperature, $T_{\mathrm{c}}$, is already at its boiling point and consequently will remain constant, to a first order approximation, during its passage through the atmosphere. $Q$ in equation (11) represents the total energy flux received by the comet surface from the densely heated gas cap in front of it. Here, we have modelled the gas cap as an outer (cooler) layer of a stellar medium. Also, $Q$ is the sum of the radiative, convective and conductive mechanisms of heat transfer as defined in the Appendix. Multiplying this energy flux by $A(M c / \rho c)^{2 / 3}$, the effective surface area of the comet, and then dividing by $L$, the latent heat of vaporization, then indicates how much mass has ablated from the comet surface. As compact as equation (11) may seem, it suffers from one important flaw : it fails to take fragmentation into account. In the case of a meteor, this can be justified since such objects are compact and structurally strong. However, in our case, a comet is a loosely held conglomerate of frozen ices and meteoritic dust and under the enormous aerodynamic stresses imposed by nature, a comet would probably fragment into many smaller pieces. Fragmentation will accelerate the ablation process as the many smaller pieces present a greater surface area than the assembled conglomerate. We will comment on this point further. Equation (11) also neglects shape variation during the comet's flight through the atmosphere.

\section{SIMULATION OF THE TUNGUSKA EVENT}

The modelling equations we employ to simulate the Tunguska Event are therefore summarized by the following:

$$
\begin{gathered}
\frac{\mathrm{d} M_{\mathrm{c}}}{\mathrm{d} t}=-\frac{A}{L}\left(\frac{M_{\mathrm{c}}}{\rho_{\mathrm{c}}}\right)^{2 / 3} Q \\
\frac{\mathrm{d} V_{\mathrm{c}}}{\mathrm{d} t}=-\frac{\Gamma A \rho_{0} V_{\mathrm{c}}^{2} \mathrm{e}^{-2 / h}}{M_{\mathrm{c}}^{1 / 3} \rho_{\mathrm{c}}^{2 / 3}}+g \cos \theta \\
\frac{\mathrm{d} z}{\mathrm{~d} t}=-V_{\mathrm{c}} \cos \theta \\
\frac{\mathrm{d} N_{\mathrm{d}}}{\mathrm{d} t}=\frac{\beta_{\mathrm{d}}}{V_{\mathrm{s}}}\left(\frac{A}{L}\right)\left(\frac{M_{\mathrm{c}}}{\rho_{\mathrm{c}}}\right)^{2 / 3} Q\left(1-L_{\mathrm{f}}\right)-N_{\mathrm{d}}^{2}(t)\langle\sigma v\rangle_{\mathrm{dd}} \\
\frac{\mathrm{d} E_{\mathrm{s}}}{\mathrm{d} t}=\bar{Q}_{\mathrm{dd}} N_{\mathrm{d}}^{2}\langle\sigma v\rangle_{\mathrm{dd}} \\
c_{\mathrm{p}} T_{\mathrm{sf}}+\frac{V_{\mathrm{c}}^{2}}{2}=c_{\mathrm{p}} T_{\mathrm{s}}+\frac{(\gamma-1) c^{2}}{4 \gamma}+\frac{\sigma T_{\mathrm{s}}^{4} \mathrm{e}^{z / h}}{\rho_{0} V_{\mathrm{c}}}
\end{gathered}
$$

In order to solve the above system of first order, nonlinear, coupled, autonomous differential equations we invoke the following preatmospheric boundary conditions at $t=0$ :

$$
\begin{aligned}
& M_{\mathrm{c}}(0)=M_{\mathrm{c} \infty} \\
& V_{\mathrm{c}}(0)=V_{\mathrm{c} \infty} \\
& z(0)=z_{\infty} \\
& N_{\mathrm{d}}(0)=N_{\mathrm{d} \infty} \\
& E_{\mathrm{s}}(0)=0 \\
& T_{\mathrm{sf}}(0)=\frac{2 T_{\mathrm{e}} \gamma(\gamma-1)}{(\gamma+1)^{2}}\left(\frac{V_{\mathrm{c} \infty}}{c}\right)^{2} .
\end{aligned}
$$

Here, we take the atmosphere to begin at $z_{\infty}=150$ $\mathrm{km}$, which implies that at this altitude the shock front is fully developed and nuclear fusion begins. The entry velocity into the Earth's atmosphere will depend upon the location of the event and the zenith angle chosen as it represents the vector sum of the Earth's orbital 
velocity $\left(30 \mathrm{~km} \mathrm{~s}^{-1}\right)$ with the comet's velocity, which is assumed to be approximately equal to the escape velocity of the Sun at the Earth's distance from the Sun (or $42 \mathrm{~km} \mathrm{~s}^{-1}$ ). It follows then that $44.6 \leqslant V_{\mathrm{c} \infty} \leqslant 57.1 \mathrm{~km} \mathrm{~s}^{-1}$ for $90^{\circ} \geqslant \theta_{\infty} \geqslant 0^{\circ}$, respectively. To determine $N_{\mathrm{d} \infty}$, we made an estimation of the mass lost along the comet's arbitrary path prior to entry into the Earth's atmosphere. However, to specify the orbit of the comet demands knowledge of both the comet's eccentricity and perihelion distance, neither of which is known. All that can be said about the Tunguska comet orbit is that it has to be retrograde as it was viewed in the early morning hours. We can infer, though, that it probably had an eccentricity close to unity as is common among comets : for example, comet Halley has an eccentricity of 0.967 . Also, an upper bound of the perihelion distance associated with the Tunguska comet can be taken to be 1 a.u. otherwise it would not have collided with the Earth. These estimates, along with a developed theory of vaporization of a comet surface have been employed in the Appendix to yield $N_{\mathrm{d} m} \approx 10^{18} \mathrm{~cm}^{-3}$ (Swamy, 1986). The theory used is in good agreement with the results from the last passage of Halley's comet (Craven et al., 1986). Lastly, we have taken the deuterium abundance to be similar to that on Earth, namely $0.0148 \%$, as we were unable to find evidence to suggest otherwise. Both $M_{\mathrm{c} \infty}$ and $\theta_{\infty}$ have been taken to be variable parameters.

\section{RESULTS}

In order to numerically integrate the system of differential equations (12)-(16), a fourth order Runge-Kutta algorithm was implemented. At each step in time, equation (17) was then numerically solved by utilizing the Newton-Raphson iterative algorithm. The values of the various constants used in the equations are listed in Table 1 . The solutions to equations (12), (13), (15), (16) and (17) are displayed in Figs 2, 3, 4, 5 and 6, respectively. These results correspond to near perpendicular entries into the Earth's atmosphere (i.e. $\mathrm{O} \leqslant \theta_{\infty} \leqslant 30^{\circ}$ ). After numerous computer simulations, it was found that the overpressure in the gas cap just prior to impact corresponding to $M_{\mathrm{c} \infty} \sim 5 \times 10^{13} \mathrm{~g}$ can best explain the flattened forest associated with this event for the zenith angles considered. This is in good agreement with existing estimates for $M_{\mathrm{c} \infty}$ (Fesenkov, 1966; Turco $e t$ al., 1982). In solving these equations, it was found that the nuclear energy released during the comet's $\sim 3 \mathrm{~s}$ passage through the atmosphere was indeed negligible. Figure 6 reveals that fusion in the stagnation volume only occurred during the last $2 \mathrm{~km}$ of the comet's trajectory where the temperature reached $4 \times 10^{5} \mathrm{~K}$ and the properties of the gas cap approached those of a fully ionized plasma. The integrated fusion energy over time and stagnation volume for a vertical entry is $\sim 10^{-4} \mathrm{~J}$, therefore suggesting that an upper limit to the total nuclear energy expenditure from the entire gas cap is $\sim 5 \times 10^{-3} \mathrm{~J}$. This enables us to conclude that if nuclear energy was liberated from the Tunguska Event, it did not result from the cometary hypothesis. Further, our simulations revealed that considerable nuclear fusion energy will only be produced when $V_{c \infty}>100 \mathrm{~km} \mathrm{~s}^{-1}$ for $M_{\mathrm{c} \infty}=5 \times 10^{13} \mathrm{~g}$. However, this entry velocity is not physical as the maximum attainable preatmospheric velocity of a meteor, as predicted by celestial mechanics, is $\sim 72$ $\mathrm{km} \mathrm{s}^{-1}$ as shown in the Appendix.

Although one should not be surprised by this finding, it was thought that the nuclear energy released would have been great enough to explain the heat felt by witnesses $60 \mathrm{~km}$ away from the point of impact. One witness described the heat radiated from the event as a sheet of Sun (Baxter and Atkins, 1976). Because of the body's brief passage through the atmosphere, the liberated heat can be viewed as originating from a cylindrical flash. The nuclear energy flux received at a distance $r$ would then be $H \sim E_{\mathrm{N}} /\left(2 \pi r t_{\mathrm{f}}\right)$. Setting $H=0.14 \mathrm{~J} \mathrm{~cm}^{-2} \mathrm{~s}^{-1}$ (i.e. solar flux), $r=60 \mathrm{~km}$ and $t_{\mathrm{f}} \sim 3 \mathrm{~s}$ yields a nuclear energy release of $E_{\mathrm{N}} \sim 16 \mathrm{MJ}$. Thus, the nuclear energy expenditure from the gas cap necessary to produce a similar heat flux as the Sun is $16 \mathrm{MJ}$. Clearly, the heat felt was not due to fusion energy, but perhaps chemical energy or the dissipated heated shock front.

\section{DISCUSSION AND CONCLUSIONS}

Our analysis thus suggests that the surviving meteor, or meteorite, spontaneously vaporized at impact leaving no noticeable crater due to its loosely held structure. A simple calculation will reveal that the energy requirement for this to occur was available. By defining

$$
Q_{\mathrm{f}}=\frac{V_{\mathrm{cf}}^{2}}{2 L}
$$

(i.e. the ratio of the total available kinetic energy at impact to the amount of energy necessary to vaporize a unit mass of the meteorite) and substituting the appropriate values, $Q_{\mathrm{f}}$ takes on the value of $\sim 550$ implying that sufficient energy to vaporize the meteorite 550 times over was available!

Immediately after impact, the strong shock front accompanying the comet through the atmosphere 
TABLE 1

\begin{tabular}{lll}
\hline Symbol & \multicolumn{1}{c}{ Meaning } & \multicolumn{1}{c}{ Value used } \\
\hline$A$ & Meteor shape factor & 1.21 \\
$g$ & Average acceleration due to gravity & $960 \mathrm{~cm} \mathrm{~s}^{-2}$ \\
$L$ & Latent heat of vaporization & $2,400 \mathrm{Jg}^{-1}$ \\
$\Gamma$ & Drag coefficient & 1.0 \\
$\sigma$ & Stefan-Boltzmann constant & $5.67 \times 10^{-12} \mathrm{~J} \mathrm{~s}^{-1} \mathrm{~cm}^{-2} \mathrm{~K}^{-4}$ \\
$T_{\mathrm{c}}$ & Comet mean temperature & $375 \mathrm{~K}$ \\
$T_{\mathrm{e}}$ & Average atmospheric temperature & $320 \mathrm{~K}$ \\
$h$ & Scale height for the assumed isothermal atmosphere & $9.6 \times 10^{5} \mathrm{~cm}^{-3}$ \\
$\rho_{0}$ & Atmospheric density at sea level & $0.0012 \mathrm{~g} \mathrm{~cm}^{-3}$ \\
$\rho_{\mathrm{c}}$ & Comet density & $1.0 \mathrm{~g} \mathrm{~cm}^{-3}$ \\
$\gamma$ & Ratio of specific heats of the gas mixture in the gas cap & $5 / 3$ \\
$c_{\mathrm{p}}$ & Specific heat at constant pressure of gas cap & $11.0 \mathrm{~J} \mathrm{~g}^{-1} \mathrm{~K}^{-1}$ \\
$c$ & Speed of sound & $3.5 \times 10^{4} \mathrm{~cm} \mathrm{~s}^{-1}$ \\
$\langle\sigma v\rangle_{\mathrm{dd}}$ & Sigma-v parameter & Empirical formula based on \\
$Q_{\mathrm{dd}}$ & Average energy release per d-d reaction & McNally et al. (1979) \\
$\beta_{\mathrm{d}}$ & Conversion factor, converting mass loss rate to corresponding gain & $5.82 \times 10^{-13} \mathrm{~J}$ \\
$V_{\mathrm{s}}$ & rate of deuterium nuclei in the stagnation volume & $7.48 \times 10^{16} \mathrm{~g}^{-1}$ \\
$L_{\mathrm{f}}$ & Stagnation volume & Varies according to (A15) \\
$Q$ & Leakage factor & Varies according to (A14) \\
\hline
\end{tabular}

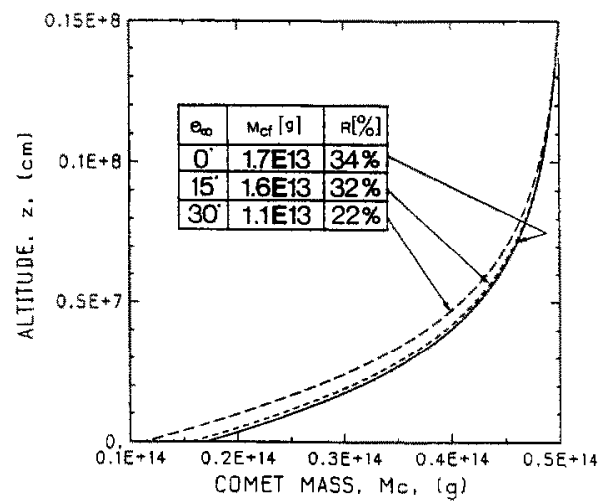

Fig. 2. MASS OF COMET VS ALTITUDE For THREE ENTRY ZENITH ANGLES.

The preatmospheric mass was taken to be $5 \times 10^{13} \mathrm{~g}$ for all three cases shown. In the figure, $M_{\mathrm{cf}}$ denotes the meteorite mass impacting the earth while $R$ is the residual mass percentage which survives the plunge through the atmosphere.

continued to propagate radially outwards into the otherwise undisturbed forest. Because of its brief passage through the atmosphere, the outward propagating disturbance can be viewed as a cylindrical shock front expanding radially, From the impact conditions, the pressure associated with the shock wave just prior to impact is dictated by

$$
P_{\mathrm{f}} \simeq \frac{2 \gamma}{\gamma+1}\left(\frac{V_{\mathrm{cf}}}{c}\right)^{2} P_{0}
$$

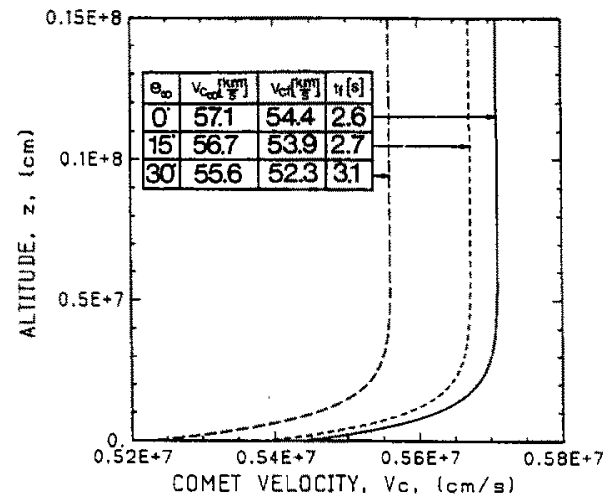

Fig. 3. COMET veloctity vs altitude for three entry ZENITH ANGLES.

All three curves clearly show that the body travels unimpeded through the atmosphere until reaching the lower stratosphere $(\sim 25 \mathrm{~km})$ where it is then quickly decelerated. In the figure, $V_{\mathrm{ef}}$ denotes the final impact velocity and $t_{f}$ depicts the flight time.

with $P_{0}$ being the atmospheric pressure at sea level. The numerical value of $P_{f}$ is of the order of 25,000 atm for $M_{\mathrm{c} \infty} \sim 5 \times 10^{13} \mathrm{~g}$. Clearly, this explains why the trees were radially knocked down and the recording of seismic and acoustic disturbances thousands of kilometres away. Assuming that the pressure decayed inversely with distance, the pressure at a distance of $30 \mathrm{~km}$ was still large enough to knock down trees. The corresponding high temperature of the gas 


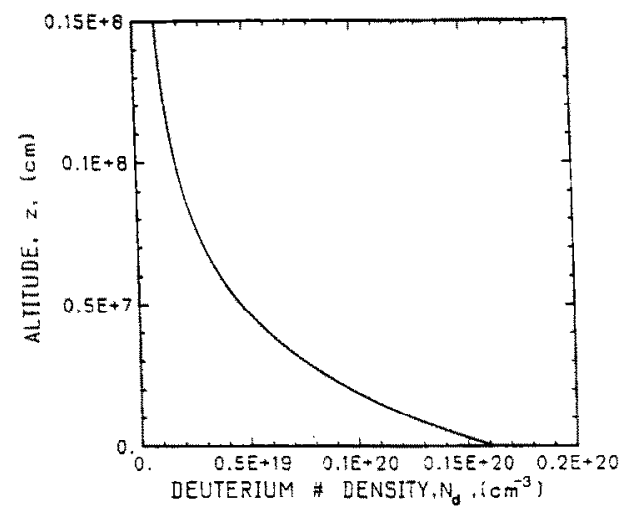

Fig. 4. Deuterium Concentration IN the stagnation VOLUME AS A FUNCTION OF ALTITUDE FOR $\theta_{\infty}=0$.

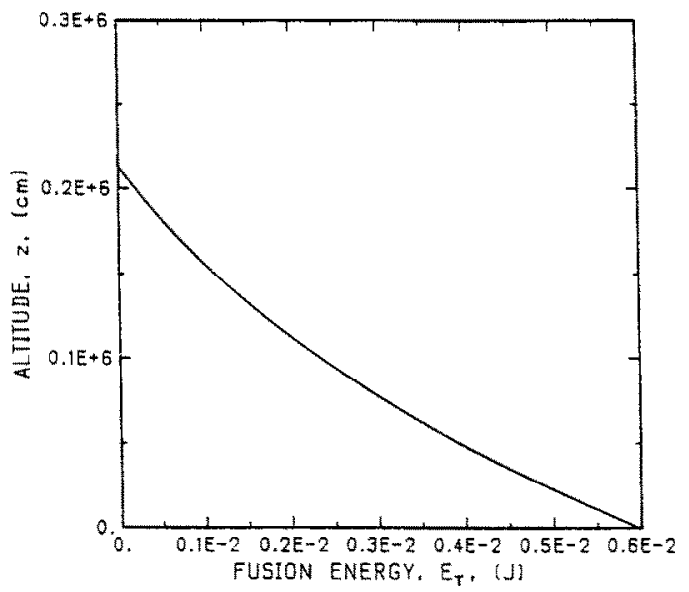

Fig. 5. TOtal NUClear fusion ENERGY liberated BY THE STAGNATION VOLUME AS A FUNCTION OF ALTITUDE FOR $\theta_{\infty}=0$. Here, $E_{\mathrm{T}}$ is obtained by multiplying the stagnation fusion energy density, $E_{\mathrm{s}}$, by the stagnation volume, $V_{\mathrm{s}}$.

cap at impact, namely $\sim 400,000^{\circ} \mathrm{C}$, quickly set the devastated forest ablaze.

The only signature the comet left behind was the brilliantly lit night skies over Europe and Asia that followed. This can be attributed to the interaction of the comet tail with the atmosphere, producing the spectacular meteor showers witnessed by many. Figure 7 is a schematic illustration of the described scenario.

We add that we have given little analytical emphasis to the observed ring of stripped upright trees within the central blasted area. This phenomenon could be explained by an explosion taking place prior to impact, several kilometers above the Earth's surface. The resulting combined effect of the explosion and ballistic waves then continued to propagate in such a complicated shape that part of the disturbance landed

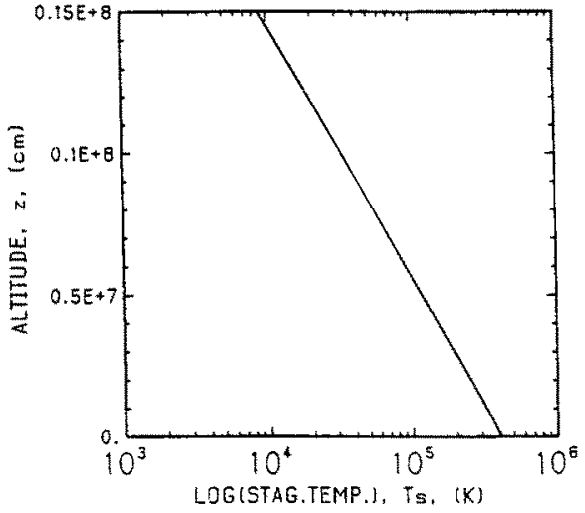

Fig. 6. Stagnation Gas CAP TEMPERATURE VS ALTITUDE FOR $\theta_{\infty}=0$.

normally over a ring of trees thus stripping and causing them to remain upright. Also, the presented model would predict that the flattened forest be symmetric, contrary to the observed peculiar form which resembles the figure of a butterfly. This shape, however, could be considered to be the result of a propagating inclined cylindrical shock front further complicated by the rough terrain over which the event took place. Some have even endeavoured to infer the entry angle from the shape of the flattened forest (Zotkin and Tsikulin, 1966; Korobeinikov et al., 1976).

We suggest that the explosion may have been triggered by mechanical destruction brought about by the enormously imposed aerodynamic pressure. This caused the comet to fragment into a dense swarm of particles which were blanketed together by a common shock wave and thus moved as a single body. The body may have then vaporized on the spot due to the sudden acceleration in ablation which accompanied the abrupt fragmentation process. In the vaporized state, gases such as methane, may have undergone violent exothermic chemical reactions.

An analogous treatment of the chemical energy released by this event has also been formulated (Park. 1978). The results claim that the associated anomalous atmospheric phenomena car be attributed to chemical reactions involving the nitric oxide produced with atmospheric ozone. It is conjectured that the produced nitric oxide fertilized the area near the fall, thus causing the observed rapid plant growth. The leaching process of $\mathrm{NO}_{2}$ by rain into the soil is held responsible.

In conclusion, we address the following thoughts.

(1) Has any radiation been registered? An expedition investigated the reported radioactivity in 


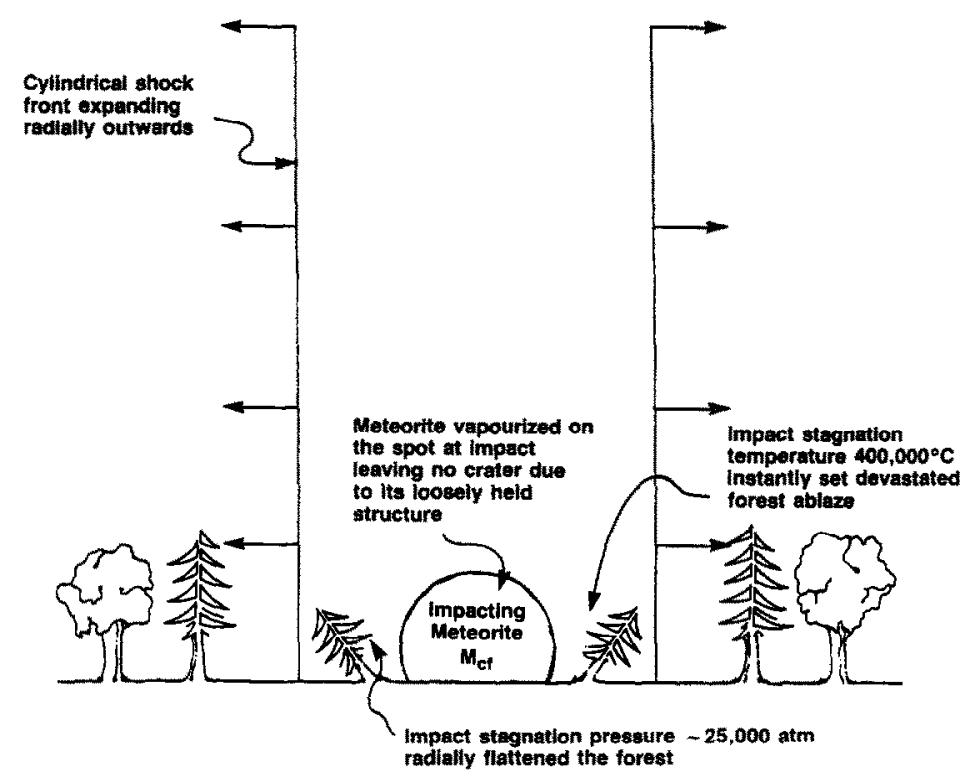

Fig. 7. SCHEMATIC ILLUSTRATION SHOWING THE POST IMPACT EFFECTS WTTH $\theta_{\infty}=0$.

deep rings of the fallen trees and established that the measured radioactivity is due to fallout from the testing of modern atomic devices which has been absorbed into the wood (Florensky, 1963). Even if our results showed that considerable nuclear energy was released, we suggest that it would not be measurable because the location of the event was not discovered until almost two decades later, at which point the existing radiation would have substantially decayed away, and also, the radiation would have been released in the atmosphere with very little reaching the Earth, and thus would be easily and quickly dissipated by atmospheric effects.

(2) Why wasn't the comet seen before reaching the Earth? Perhaps the observing techniques were not as extensive, and as trivial as they are today. Also, it has been proposed that the comet may have been a fragment of a larger comet (believed to be comet Encke) that dislodged itself from the parent comet at the last moment (Kresak, 1978). Although the parent comet may have been tracked, the dislodged fragment was not.

(3) Why were no remains of the meteorite recovered? An expedition conducted in 1962 (Florensky, 1963) recovered a concentration of meteoritic dust $60-80 \mathrm{~km}$ Northwest of the believed epicenter, prompting the claim that this find promotes the meteor hypothesis over the comet hypothesis. We suggest that this find shows no conclusive evidence pointing towards the meteorite hypothesis as comets con- tain meteoritic dust embedded in it ; indeed, the other materials are volatile and thus would leave no trace. The observation that the dust was recovered some distance from the epicenter supports the hypothesis that the object exploded in flight. Our model would have to admit that some meteoritic dust should have been left behind in the immediate impact area.

(4) Extrapolating our findings to other planets in the solar system, we suggest that a similar event occurring on Venus will yield a significantly larger amount of fusion energy. Reasons for believing so are the following: the maximum entry velocity the comet may possess is $85 \mathrm{~km} \mathrm{~s}^{-1}$ implying higher temperatures in the gas cap, and the atmospheric pressure, density and temperature are much greater than that of Earth causing ablation to be more severe and thus supplying more deuterium. Also, the atmosphere of Venus is rich in hydrogen when compared with the Earth, therefore providing an even higher dueterium concentration to result in the gas cap. Lastly, the preatmospheric deuterium concentration will be significantly higher as the comet will have lost more mass in travelling the extra distance to Venus.

Acknowledgements-Financial support for this research has been provided by the Natural Sciences and Engineering Research Council of Canada.

\section{REFERENCES}

Ahrens, T. J. and O'Keefe, J. D. (1987) Impact on the Earth, ocean and atmosphere. Int. J. Impact Engng 5, 13. 
Alfvén, H. and Arrhenius, G. (1975) Structure and Evolutionary History of the Solar System. D. Reidel, Dordrecht.

Baldwin, B. and Sheaffer, Y. (1971) Ablation and breakup of large meteoroids during atmospheric entry. $J$. geophys. Res. 76, 4653.

Baxter, J. and Atkins, J. (1976) The Fire Came By. Doubleday and Company Inc., Garden City, New York.

Biberman, L. M., Bronin, S. Ya. and Brykin, M. V. (1980) Moving of a blunt body through the dense atmosphere under conditions of severe aerodynamic heating and ablation. Acta Astronautica 7, 53.

Bronshten, V. A. (1965) Problems of Motion of Large Meteoritic Bodies in the Almosphere. Rand Corporation, U.S.A.

Chen, F. C. (1974) Introduction to Plasma Physics. Plenum Press, New York.

Cowan, C., Atluri, C. R. and Libby, W, F. (1965) Possible anti-matter content of the Tunguska meteor of 1908. Nature 206, 861.

Craven, J. D. et al. (1986) The hydrogen coma of comet Halley before periheilion: preliminary observations with Dynamics Explorer 1. Geophys. Res. Lett. 13, 873.

Crowther, J. G. (1931) More about the great Siberian meteorite. Scient. Am. 144. 314.

Fay, J. A., Moffatt, W. C. and Probstein, R. F. (1963) An analytical study of meteor entry. AIAA Conf. on Physics of Entry into Planetary Atmospheres. M.I.T., Cambridge, Massachusetts.

Fesenkov, V. G. (1966) A study of the Tunguska meteorite fall. Soviet Astr. 10, 195.

Florensky, K. P. (1963) Did a comet collide with the Earth in 1908? Sky Telescope 25, 268.

Freeman, N. C. (1956) On theory of hypersonic fiow past plane and axially symmetric bluff bodics. $J$. fluid Mech. 1 , 366.

Gill, R. D. (Ed.) (1981) Plasma Physics and Nuclear Fusion Research. Academic Press, London.

Goulard, R. (1964) Preliminary estimates of radiative transfer effects on detached shock layers. $A I A A J .2,494$.

Harms, A. A. (1987) Formulation of self fusion reaction rate expression. Aromkernergie-Kerntechnik 49, 168.

Jackson, A. A., IV and Ryan, M. P. (1973) Was the Tungus Event due to a Black Hole? Nature 245, 88.

Kresak. L. (1978) The Tunguska object-part of comet Encke? Bull. astr. Insts Czech. 29, 129.

Korobeinikov, V. P., Chuskin, P. I. and Shurshalov, L. V. (1976) Mathematical model and computation of the Tunguska meteorite explosion. Acta Astronautica. 3, 615.

McNally, J. R., Jr., Rothe, K. E. and Sharp, R. D. (1979) Fusion Reactivity Graphs and Tables for Charged Particle Reactions. Prepared by the Oak Ridge National Laboratory.

Oberg, J. E. (1977) Tunguska: collision with a comet. Astro. $5,18$.

Oliver, C. P. (1928) The great Siberian meteorite. Scient. Am. $139,42$.

Öpik, E. J. (1958) Physics of Meteor Flight in the Atmosphere. Interscience, New York.

Park, C. (1978) Nitric oxide production by Tunguska meteor. Acta Astronautica 5, 523.

Silver, L. T. and Schultz, P. H. (Eds) (1981) Geological implications of impacts of large asteroids and comets on the Earth. Special Paper \# 192, The Geological Society of America.

Swamy, K. (1986) Physics of Comets. World Scientific, New York.
Turco, R. P. et al. (1982) An analysis of the physical, chemical, optical and historical impacts of the 1908 Tunguska meteor fall. Icarus $\mathbf{5 0}, 1$.

Whipple, F. J. (1930) The great Siberian meteor and the waves it produced. $Q . J l R$. met. Soc. (London) 16, 287.

Witze, P. O. and Dwyer, H. A. (1976) The turbulent radial jet. J. Fluid Mech. 75, 401 .

Zotkin, I. T. and Tsikulin, M. A. (1966) Simulation of the explosion of Tungus meteorite. Sov. Phys. Dokl. 11, 183.

\section{APPENDIX}

Entry velocity into atmosphere

Most texts on celestial mechanics assert that the relative velocity, $V$, of a body of mass $M_{\mathrm{c}}$, with respect to the Sun, of mass $M_{\text {SUN }}$, is governed by the energy equation

$$
V^{2}=G\left(M_{\text {SUN }}+M_{\mathrm{c}}\right)\left(\frac{2}{r}-\frac{1}{a}\right)
$$

where $G$ is the universal gravitational constant, $r$ is the distance of separation and $a$ is the length of the semi-major axis. Because comets are generally believed to travel along near parabolic heliocentric orbits, the semi-major axis approaches infinity. Thus, neglecting the perturbations from the other planets, the relative velocity of the comet evaluated at $r=1$ a.u. (i.e. when it crosses the Earth's orbit) with $M_{\mathrm{c}} \ll M_{\text {SUN }}$ gives $V \doteq 42 \mathrm{~km} \mathrm{~s}^{-1}$. This velocity must be vectorially added with the Earth's orbital velocity of $\sim 30 \mathrm{~km}$ $\mathrm{s}^{-1}$. Limiting cases occur when: a meteoroid overtakes the Earth and approaches at a speed of $42-30$ or $12 \mathrm{~km} \mathrm{~s}^{-1}$, or if it meets the Earth in a head-on collision and approaches at a speed of $42+30$ or $72 \mathrm{~km} \mathrm{~s}^{-1}$.

Heat transferred from gas cap to comet stagnation surface

The total energy flux received by the comet surface from the gas cap is comprised of three contributions: radiation, convection and conduction (solar radiation no longer contributes as it is blocked by the gas cap).

For an opaque gas, the radiation heat flux will be that of continuum diffuse radiation given by Fay et al. (1963):

$$
q_{\mathrm{R}}=-\frac{16}{3} \sigma T_{\mathrm{s}}^{3} I_{\mathrm{R}}\left(\frac{\mathrm{d} T_{\mathrm{s}}}{\mathrm{d} y}\right)_{\mathrm{w}}
$$

where $l_{\mathrm{R}}$ is the Rosseland mean free path and $\left(\mathrm{d} T_{s} / \mathrm{d} y\right)_{\mathrm{w}}$ is the temperature gradient evaluated at the comet surface. For free-free and free--bound transitions of electrons, the Rosseland mean free path is given by

$$
l_{\mathrm{R}}=\frac{45}{32 \pi^{4}} \frac{m_{\mathrm{e}} c\left(k T_{\mathrm{s}}\right)^{3}}{n_{\mathrm{i}} n_{\mathrm{c}} \bar{Z}_{\mathrm{i}} \mathrm{e}^{6} h^{2}}\left(\frac{6 m_{\mathrm{e}} k T_{\mathrm{s}}}{\pi}\right)^{1 / 2} \int_{0}^{\infty} \frac{x^{7} \mathrm{e}^{x}}{\left(\mathrm{e}^{x}-\mathrm{l}\right)^{3}} \mathrm{~d} x .
$$

Here, $m_{\mathrm{e}}$ is the electron mass, $n_{\mathrm{i}}$ and $n_{\mathrm{e}}$ are the ion and electron number densities, respectively, $\mathrm{e}$ is the electron charge, $c$ is the speed of light, $k$ is the Boltzmann constant, $h$ is Planck's constant and $Z_{\mathrm{i}}$ is the average ionic charge. The approximate numerical value of the integral is 22.6 (dimensionless).

The convective heat flux to the comet surface is given by Fay et al. (1963):

$$
q_{\mathrm{c}}=\left\{\frac{2 \sqrt{2} V_{\mathrm{c}} \sqrt{\rho_{\mathrm{a}} \rho_{\mathrm{s}}} k_{\mathrm{e}} c_{\mathrm{p}}}{R_{\mathrm{c}}}\right\}^{1 / \rho} T_{\mathrm{s}}\left[\theta^{3 / 2} \frac{\mathrm{d} \theta}{\mathrm{d} n}\right]_{\mathrm{w}}
$$


where $\rho_{\mathrm{a}}$ is the air density, $\rho_{\mathrm{s}}$ is the stagnation gas cap density, $R_{\mathrm{c}}$ is the comet radius, $c_{\mathrm{p}}$ is the specific heat of the gas and $k_{\mathrm{e}}$ is the thermal conductivity. The dimensionless quantity $\left[\theta^{3 / 2}(\mathrm{~d} \theta / \mathrm{d} n)\right]_{\mathrm{w}}$ has a value of about 0.38 (Fay et al., 1963). The thermal conductivity is given by Spitzer's expression for a fully ionized plasma

$$
k_{\mathrm{c}}=40\left(\frac{2}{n}\right)^{3 / 2} \frac{k\left(k T_{\mathrm{s}}\right)^{5 / 2}}{m_{\mathrm{e}}^{1 / 2} \mathrm{e}^{4} \bar{Z}_{\mathrm{i}} L}
$$

wherc $L$ is the Coulomb logarithm given by

$$
L=\ln \left(1+\Lambda^{2}\right)
$$

where

$$
\Lambda=\frac{3\left(k T_{s}\right)^{3 / 2}}{2 \sqrt{\pi} \mathrm{e}^{3} n_{\mathrm{e}}^{1 / 2} \bar{Z}_{\mathrm{i}}} .
$$

The conductive heat flux will be mostly due to electrons as opposed to ions because the thermal velocity of electrons will be $\sqrt{m_{\mathrm{i}} / m_{\mathrm{e}}} \sim 200$ times greater than that for ions (assuming that the electrons and ions are both at temperature $T_{\mathrm{s}}$. Thus,

$$
q_{\mathrm{c}}=-k_{\mathrm{e}}\left(\frac{\mathrm{d} T_{\mathrm{s}}}{\mathrm{d} y}\right)_{\mathrm{w}} .
$$

In expressions (A2) and (A8), the temperature gradient at the surface was estimated by

$$
\left(\frac{\mathrm{d} T_{s}}{\mathrm{~d} y}\right)_{\mathrm{w}} \sim-\frac{\left(T_{\mathrm{s}}-T_{\mathrm{b}}\right)}{\Delta}
$$

where $T_{\mathrm{b}}$ is the temperature of the comet surface assumed to be at its boiling point and $\Delta$ is the thermal boundary layer thickness given by

$$
\Delta=\frac{\delta}{\sqrt{\operatorname{Re} \cdot \operatorname{Pr}}}
$$

with $\operatorname{Re}$ as the Reynolds' number and Pr being the Prandtl number. Again, $\delta$ is the stand-off distance. Thus, the total heat flux to the comet surface is given as

$$
Q=q_{\mathrm{R}}+q_{\mathrm{c}}+q_{\mathrm{e}}
$$

with convection as the dominant mechanism for heat transfer.

\section{Stagnation volume thermodynamics and leakage}

It has been assumed that both the electrons and ions arc in equilibrium at the same temperature, $T_{\mathrm{s}}$. The thermodynamic properties of the gas are those corresponding to a fully ionized plasma. Thus, the specific heat was found from

$$
c_{\mathrm{p}}=\frac{5}{2}\left(\frac{k}{m}\right)\left(1+\bar{Z}_{\mathrm{i}}\right)
$$

with $k$ as the Boltzmann constant and $m$ as the mean molecular weight of the gas. Also, the equation of state was taken to be the perfect gas law. Lastly, photons are taken to be subject to Planck statistics, while all particles obey Maxwellian statistics.

It is recognized that the thermodynamic properties will vary along the trajectory; however, in the lower stratosphere, the properties will approach the idealized ones listed above. A criterion by which the validity of the perfect gas law can be judged is by the comparison of the Debye radius, $\lambda_{D}$, with the average distance between neighbouring particles. A necessary condition that will enable one to neglect the interactions between the gas particles - as is the case in a perfect gas-is that $\lambda_{\mathbf{D}}$ be greater than the average distance between neighbouring particles. In our case, both quantities are of the same order of magnitude.

To estimate the leakage of mass from the stagnation volume, we suggest modelling this as the impingement of two directly opposed radial jets as depicted in Fig. A1. Although an analytical solution to this problem does not exist, empirical relations based on experimental findings are available (Witze and Dwyer, 1976). The velocity profile of the escaping gas, according to experimental results, has the form

$$
V(y)=\bar{V}\left(\frac{6.226 \Delta}{r_{\mathrm{s}}}\right)^{1 / 2} \operatorname{sech}^{2}\left(8.311 y / r_{\mathrm{s}}\right) .
$$

Here, $V$ is the velocitiy at which mass ablates from the comet's surface, assumed to correspond to the mean Maxwellian velocity. Integrating the escaping mass over a cylindrically shaped stagnation surface and dividing by the mass influx, $\pi r_{\mathrm{s}}^{2}\left(\rho_{\mathrm{a}} V_{\mathrm{c}}+\rho_{\mathrm{v}} V\right)$, yields the following expression for the normalized leakage factor:

$$
L_{\mathrm{f}}=0.6\left(\frac{\Delta}{r_{\mathrm{s}}}\right)^{1 / 2}\left(\frac{\rho_{\mathrm{s}} \bar{V}}{\rho_{\mathrm{v}} \overline{\bar{V}}+\rho_{\mathrm{a}} V_{\mathrm{c}}}\right) .
$$

Here, $\rho_{\mathrm{v}}$ is the density of the ablating vapour, $r_{\mathrm{s}}$ is the radius of the stagnation volume and $\tanh \left(8.311 \delta / r_{s}\right)$ justifiably taken as unity. The stagnation volume, $V_{s}$, was estimated by

$$
V_{\mathrm{s}} \simeq \pi r_{\mathrm{s}}^{2} \delta
$$

with

$$
r_{\mathrm{s}}=\frac{R_{\mathrm{c}}}{8}
$$

and for a hypersonically travelling blunt body, the stand-off distance, $\delta$, can be shown to be (Freeman, 1956)

$$
\delta \simeq \frac{\gamma-1}{\gamma+1} R_{\mathrm{c}} .
$$

The only weakness in this interpretation of leakage is associated with (A13), which represents the fully developed velocity profile, though it is used in a regime where the flow field is not fully developed.

\section{Preatmospheric vaporization of comet nucleus}

As a comet approaches the Sun in an assumed highly elliptical orbit, its heliocentric distance will at first vary slowly thus allowing the nucleus surface to reach a steady stale temperature which is also slowly varying. Further assumptions include a slowly rotating nucleus and the neglecting of conduction. The temperature distribution on the sunlit face can then be determined by an energy halance. The fraction of solar energy absorbed must be equal to the latent energy used to transform the frozen surface to vapour plus the energy reradiated back to space. This steady state situation can be mathematically stated as follows (Swamy, 1986)

$$
F_{0}\left(1-A_{0}\right) \frac{1}{r^{2}} \cos \alpha=\sigma\left(1-A_{1}\right) T^{4}+\mathcal{Z}(T) L(T)
$$

where the symbols represent the following :

$F_{0}$ : solar flux at 1 a.u.,

$A_{0}$ : nucleus albedo in the visible,

$\alpha$ : angle that surface area makes with the impinging solar flux,

$A_{1}$ : nucleus albedo in the infrared,

$\sigma$ : Stefan-Boltzmann constant, 


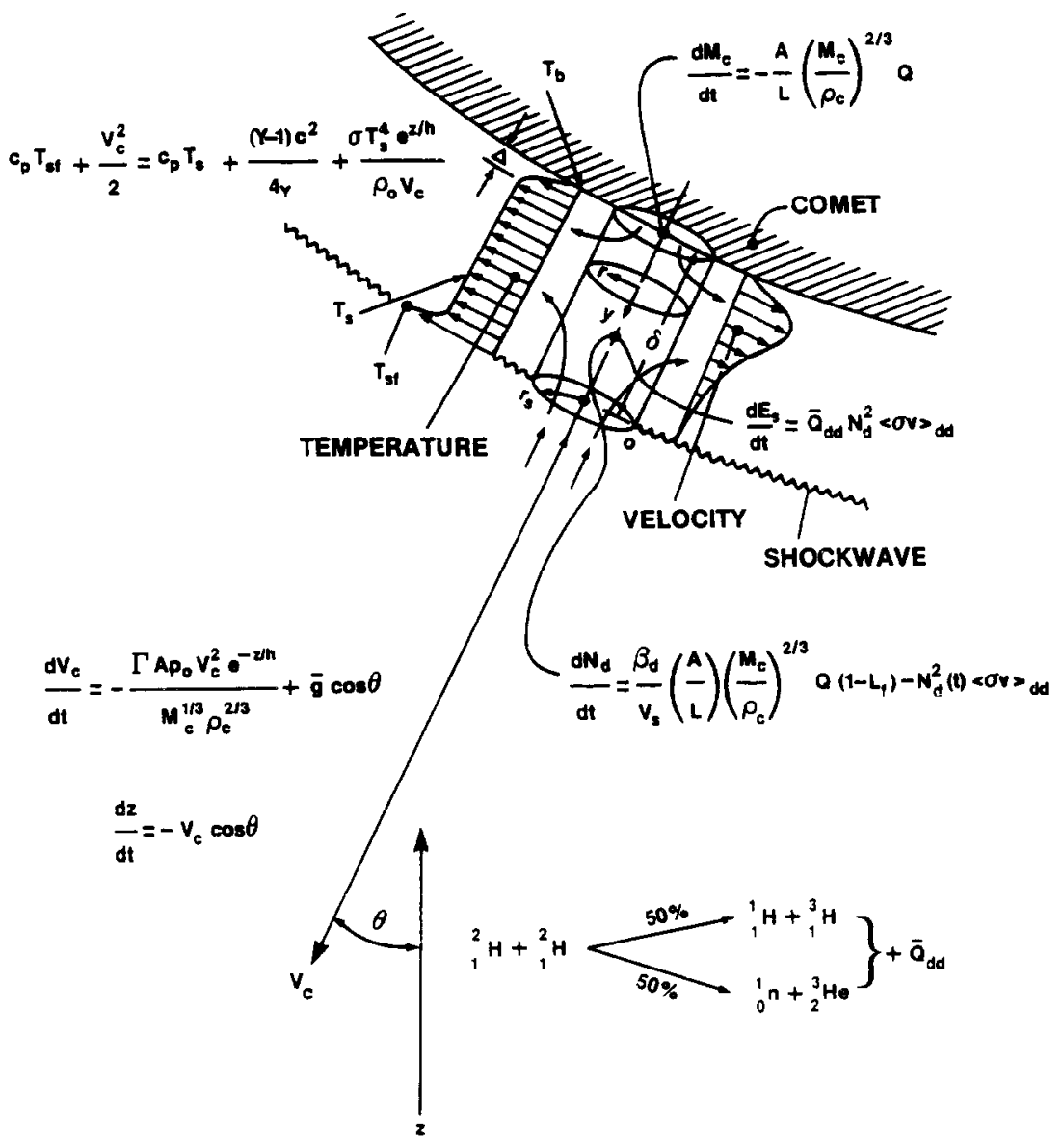

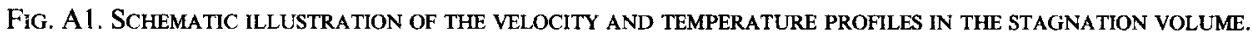
Not included here are the effects of the blowing layer formed adjacent to the temperature profile; it is judged that in the lower atmosphere, this will be of little consequence.

$Z(T)$ : the production rate of vapour (in molecules $\mathrm{cm}^{-2}$ $\mathrm{s}^{-1}$ ),

$L(T)$ : the latent heat energy per molecule for the vaporization of the surface.

If equilibrium is achieved, then

$$
Z(T)=\frac{P}{(2 \pi m k T)^{1 / 2}}
$$

where $P$ is the vapour pressure.

Simultaneously solving (A18) and (A19) iteratively will yield the unknowns $Z(T)$ and $T$, for specified values of the remaining parameters. By integrating $Z$ along the comet's assumed orbit, an estimation as to how much mass has ablated away can be made. It has been suggested that the form of the solution can be rendered as in Swamy (1986)

$$
Z(r)=Z_{0} \alpha\left(\frac{r}{r_{1}}\right)^{-m}\left\{1+\left(\frac{r}{r_{1}}\right)^{n}\right\}^{-k} .
$$

Taking the comet to be chiefly composed of $\mathrm{H}_{2} \mathrm{O}$, as found in the last passage of comet Halley (Craven et al., 1986), then $r_{1}=2.808$ a.u., $m=2.15, \quad n=5.093, k=4.6142$, $\alpha=0.111$ and $Z_{0}=1 / 7 \times 10^{18}$. Also, based on Newtonian mechanics

$$
r(\theta)=r_{0}\left(\frac{1+e}{1+e \cos \theta}\right)
$$

where $r_{0}$ is the perihelion distance (assumed to occur at $\theta=0$ ) and $e$ is the eccentricity. Further,

$$
\theta=\frac{1}{r^{2}} \sqrt{r_{0}(1+e)} G \overline{M_{\mathrm{SUN}}} .
$$

Utilizing (A20)-(A22), the integrated vaporized mass becomes

$$
\begin{aligned}
\int_{r_{0}}^{r_{1}} Z \mathrm{~d} t=\int_{r_{0}}^{r_{1}} Z\left(\frac{\mathrm{d} t}{\mathrm{~d} \theta}\right) & \left(\frac{\mathrm{d} \theta}{\mathrm{d} r}\right) \mathrm{d} r=\frac{\sqrt{r_{0}(1+e)}}{e \sqrt{G M_{\mathrm{SUN}}}} \\
& \times \int_{r_{0}}^{r_{1}} \frac{Z(r)}{\sqrt{1-\left[\frac{r_{0}(1+e)}{e r}-\frac{1}{e}\right]^{2}}} \mathrm{~d} r
\end{aligned}
$$


Assuming that the comet orbit had $e \sim 0.8$ and $r_{0} \sim 1$ a.u., numerical evaluation of (A23) gives $\sim 10^{25}$ molecules of $\mathrm{H}_{2} \mathrm{O}$ per centimetre. Thus, the preatmospheric deuterium concentration is

$$
N_{\mathrm{d} \infty} \sim 2 \times 10^{25} \times(0.000148)\left(\frac{1}{\delta}\right) \simeq 10^{18} \mathrm{~cm}^{-3} .
$$

We also estimate that the gas cap density at entry, $\rho_{\mathrm{s} \infty}$, is $\sim 0.2 \mathrm{~g} \mathrm{~cm}^{-3}$, for the case that the shock front is fully developed. Lastly, result (A24) neglects losses brought about by solar radiation and solar flares which cause dust and ion tails to form.
As a general concluding point on the methodology employed here, we may justifiably claim that the trajectory and other dynamical considerations employed here, follow as a logical consequence of physical modelling which has developed in the literature over the years (Opik, 1958; Bronshten, 1965; Baldwin and Sheaffer, 1971; Biberman et al., 1980); nuclear reaction considerations introduced here correspond to recent developments in plasma and fusion physics. Finally, issues of the terrestrial impact of large asteroids and comets are outside our area of interest but information on this subject is readily available (Silver and Schultz, 1981; Ahrens and O'Keefe, 1987). 